\title{
Germanica
}

\section{Heiner Müllers Ge(spenste)rma-nia Germania 3 Gespenster am Toten Mann}

André Combes

\section{OpenEdition}

1 Journals

Édition électronique

URL : http://journals.openedition.org/germanica/2334

DOI : 10.4000/germanica.2334

ISSN : 2107-0784

Éditeur

Université de Lille

\section{Édition imprimée}

Date de publication : 30 décembre 1999

Pagination : 59-75

ISBN : 9782913857018

ISSN : 0984-2632

\section{Référence électronique}

André Combes, « Heiner Müllers Ge(spenste)rma-nia Germania 3 Gespenster am Toten Mann »,

Germanica [Online], 25 | 1999, Online erschienen am: 30 Januar 2014, abgerufen am 06 Oktober 2020

URL : http://journals.openedition.org/germanica/2334; DOI : https://doi.org/10.4000/germanica.2334

Ce document a été généré automatiquement le 6 octobre 2020.

(c) Tous droits réservés 


\title{
Heiner Müllers Ge(spenste)rma-nia Germania 3 Gespenster am Toten Mann ${ }^{1}$
}

\author{
André Combes
}

Die Toten warten auf der Gegenschräge /

Manchmal halten sie eine Hand ins Licht / Als lebten sie. Bis sie sich ganz zurückziehen / In ihr gewohntes Dunkel das uns blendet.

Heiner Müller²

\section{Manisches Germanisches}

Das mythologische Fundament der Stücke, die zu einer Germania-Trilogie vereinigt werden können - Die Schlacht (1951-1974), Germania Tod in Berlin (1956/71), Germania 3 Gespenster am Toten Mann (1995/96) - bildet der seit den antinapoleonischen Befreiungskriegen virulent gewordene Germania-Topos - man denke nur an Kleists Hermannschlacht (1808) -, der als ideologischer Katalysator deutscher BefreiungsEinigungs- und Herrschaftsdiskurse, im phantasmatischen Gebilde einer von Hitler erträumten und von Speer teilweise realisierten großdeutschen Hauptstadt des Dritten Reichs kulminierte. Dieser verhängnisvolle Topos wird bei Müller nicht nur als kriegerische Allegorie des ewig geteilten und geschundenen deutschen «Volkskörpers» inszeniert, der auf Biegen und Brechen in seiner einheitlichen germanischen Urkraft und gegen alles Fremde wiederherzustellen sei. Mit verarbeitet wird die komplementäre dunkle Mythologie des Nibelungenlieds, das nicht von ungefähr 1810 zum ersten Mal gedruckt wurde. In Germania Tod in Berlin wurde «Germania » eindeutig zur symbolischen "bösen Mutter », die als großdeutsche Hebamme das von Goebbels geborene Hitler-Kind - einen vieldeutigen «Contergan-Wolf» - zur Welt brachte. In ihrer aktualisierten Form galt sie Müller als « Verkörperung des deutschen Volkes in der Gestalt einer Frau im Waffenschmuck, ein Land der Krieger wie das alte Rom, ein Volk der Selbstzerfleischung wie die Nibelungen, ein Volk in Waffen, an denen es am Ende zugrunde geht: Tod im (gespaltenen) Berlin ${ }^{3}$.» Im Mittelpunkt des Stücks stand bezeichnenderweise - als eine Art «Urszene» der Ent-stehung deutscher 
Territorialität - Tacitus' Germania-Stelle über den Kampf der von der Weser getrennten " feindlichen Brüder ", Arminius und Flavius, die die von der Elbe getrennten deutschdeutschen «feindlichen Brüder» der Nachkriegszeit metaphorisch vorwegnahm. Als wäre diese kanonische Erzählung die Umwandlung wirklicher Geschichte in einen negativen Gründungsmythos gewesen, der ein immer wiederkehrendes Grundmuster fataler deutscher Identität prägte und deutsche Geschichte lediglich als ewige Wiederkehr eines katastrophalen Gleichen erscheinen ließ. Die sich seit der germanischen grauen Vorzeit zerfleischenden deutschen Brüder - Arminius und Flavius (Germania Tod in Berlin), Stalingrad-Soldaten und ihr mythologisches Pendant (Nibelungen), die ihresgleichen "fressen» (Germania Tod in Berlin, Germania 3), Kommunist und Nazi (Die Schlacht, Germania Tod in Berlin), sogar in Personalunion (Szene 3, Teil 3 von Germania 3) -, alle stammen von derselben mythischen GermaniaMutter, in deren Namen geographische und politische Teilungen wie Einigungen bzw. Wiedervereinigungen als Wiederholungszwang zum mörderischen und selbstmörderischen Zwist in jeweils anderer Gestalt vollzogen werden. Das anthropologische Grundmuster dazu mag Carl Schmitts erzkonservative Freund/FeindMythologie geliefert haben, dessen Ex Captivitate Salus (1950) in BEA zitiert wird : « Und wer kann mich wirklich in Frage stellen ? Nur ich mich selbst. Oder mein Bruder. [...]. Der Andere ist mein Bruder [...] und der Bruder erweist sich als mein Feind ${ }^{4}$. »

2 Müllers Darstellung der deutschen Geschichte erschöpft sich aber nicht im Inszenieren der variierten Modalitäten eines sinnlosen gegenseitigen Abschlachtens von «feindlichen Brüdern", die vom jeweiligen " großen Bruder » (Cäsar, Napoleon oder Stalin) fremdgesteuert würden. In den Germania-Stücken soll eher gezeigt werden, wie die von den Anfängen der preußischen Militärmonarchie datierende Deformierung der menschlichen Affekte $^{5}$ und die Produktion / Reproduktion faschistoider Verhaltensmuster ein tragisches Kontinuum darstellen, das politische Herrschaftssysteme zu deren Stabilisierung brauchen (Preußen, Deutsches Reich, NSStaat) oder wider besseres Wissen reproduzieren (stalinisierter DDR-Sozialismus) ${ }^{6}$. Dennoch konnte Müller 1979 noch erklären, er hoffe auf eine Welt, « in der Stücke wie GERMANIA TOD IN BERLIN nicht mehr geschrieben werden können, weil die Wirklichkeit das Material dafür nicht mehr bereithält $»^{7}$. Zwei Jahrzehnte später hielt die deutsche Wirklichkeit anscheinend genug « Material » zu diesem Thema bereit, um eine neue dramaturgische Bearbeitung des Germania-Mythos zu rechtfertigen. Gezeigt werden sollte diesmal, daß die letzte staatliche Germania-Gestalt - eine um die ehemalige DDR erweiterte BRD - ein eher peripheres Ereignis darstellte. So wird sie auch in Germania 3 keiner (ganzen) Szene gewürdigt. Und darin liegt die eigentliche paradoxe Aktualität des Textes: ein Nachvereinigungsstück zu sein, in dem die «Wende» als geschichtlicher Zwischenfall kaum erwähnenswert erscheint. Als sollte sie nur den Anlaß dazu liefern, angesichts des nun freiliegenden Scherbenhaufens des "realexistierenden Sozialismus" ein neues Licht auf einige der signifikantesten Epochen und Schauplätze der an Katastrophen reichen Vorgeschichte der "Vereinigung », d.h. auch der Teilung, zu werfen".

3 In diesem Zusammenhang wird die vereinigte Germania zwar nicht explizit in die lange Kette der deutschen Katastrophen eingereiht aber auch nicht als deren Unterbrechung gezeigt, sondern eher als Neuanfang im Geiste der ewigen Wiederkehr eines Kapitalismus, der nicht das Ende des deutschen Sozialismus, sondern höchstens das Ende des « realexistierenden » bedeutete. Die Ereignisse der Jahre 1989-90 haben nur 
die offizielle Bankrotterklärung nach sich gezogen. Denn der usprüngliche Sozialismus, von dem man noch « in Buchenwald und in Spanien » träumen konnte, so Thälmann zu Ulbricht in der ersten Szene, war an sich selbst gescheitert. " Hier liegt er begraben ", sagt Thälmann weiter, und zeigt auf dessen "Mausoleum», die Berliner Mauer". Thälmanns Fazit, wohlgemerkt ohne Fragezeichen : «Was haben wir falsch gemacht ${ }^{10}$. » Die Wende brachte aber auch die Wiederkehr des Kapitalismus, so Müllers Darstellung, als Sieg des alten Neuen in seiner ganzen Ambi-guität zum Ausdruck. Sie wird in einer einzigen Teilszene (Sz. 5, Teil 3) als eine Art sanfter Schrecken im Geiste der wirtschaftspolitischen Revanche-Formel «Rückgabe vor Entschädigung inszeniert. Als zynischer Pseudofortschritt «vom Blutadel zum Geldadel» (S.43-44), der - ein Novum in der deutschen Geschichte - das ewige Spuken des eigentlichen Schreckens dadurch verdrängt, daß er es vermarktet: das "Gespensterschloß bei Parchim », das seit dem deutschen Zusammenbruch leersteht, soll von den späten Erben - zwei jungen Männern in « Maßanzügen, Mode 1990 » - in einen Reiterhof « für die neue Aristokratie aus Hamburg » verwandelt werden (S. 43). Daß jedoch der wahre Schrecken das dramaturgische Feld noch nicht geräumt hat, zeigt dessen aktuellste Neuauflage. Diese wird als scheinbar selbständige monologische Texteinlage an der Gestalt des «Kroaten » verdeutlicht : als ehemaliger SS-Mann bäuerlicher Abkunft, der 1945 drei Generationen von deutschen Offizierswitwen mit dem Beil tötet - daher das «Gespensterschloß » zitiert er die faschistische Ustascha-Bewegung, als zeigenössischer Gastarbeiter, der diesmal in aller Ruhe und scheinbar völlig grundlos seine eigene Familie umbringt und dann zurück in die BRD fährt, den Bosnienkrieg 1996. Der betont sachlich-neutrale Monolog unterstreicht das völlig Mechanische am gefühllos und kaltblütig ausgeführten Mord an Frau und Kindern und inszeniert die Wiederkehr des Grauens als aktuellste Form der «Banalität des Bösen» (H. Arendt), die sich am Rande des kapitalistischen Alltags abspielt: "Mit meinen Händen, die zwei Jahre lang in Deutschland am Fließband gearbeitet haben, töte ich meine Kinder. Ich verlasse das Haus. Ich schließe die Tür ab und werfe den Schlüssel weg, [...] und fahre zurück nach Deutschland.» (S. 42). Es ist wahrscheinlich kein Zufall, wenn diese Szene in der Szenenfolge genau an zentraler Stelle steht.

\section{Prolog im Archiv : die Produktivkraft der Phantasmagorien}

5 Die Wende von 1989-1990 war darüber hinaus für Müller eine Befreiung der besonderen Art. Seiner nur scheinbar paradoxen Grundüberzeugung, daß die Allmacht des Dokuments die literarische Imagination eher befreit als behindert, verdanken wir die Entstehung von Germania 3: "Die Öffnung der Archive nach dem Zusammenbruch der DDR war Heiner Müllers Argument für seine Rückkehr zum Drama. Wenn alles Dokumentarische offenliege, sagte er im Sommer 1990, könne man als Autor wieder anfangen, Stalin und Hitler zu erfinden ${ }^{11}$. » Immer schon wollte Müller die Fiktion vom Dokument befreien, was auch damit zu tun hatte, daß er sich zeitlebens gegen die " Aushöhlung von Geschichtsbewußtsein durch einen platten Begriff von Aktualität » verwahrte $^{12}$. Erlösend wirkte also die Öffnung der Archive insofern, als sie dem Recht des Dichters auf einen fiktionalen Zugang zur Realität zu ihrem einzig angemessenen, sprich literarischen Ausdruck verhalf. Es geht bei Müller um die ErFindung der historischen Wahrheit als Fabrikation von sinnvollen Fiktionen - u.a. durch 
Verarbeitung schon bestehender - und nicht um deren « objektive ", d.h. letztendlich naturalistische Findung, wie bei den Berufshistorikern. Insofern ist die Nacht der Archive den individuellen und kollektiven Phantasmagorien zuträglich, die den Müllerschen Fiktionen als Stoff- und Topoïlieferanten dienen. Denn Geschichte, zumal die deutsche, ist ihm primär Überlagerung von dokumentarisch belegbarem Geschehen durch subjektive Assoziationen, die aus den unterschiedlichsten Manifestationen des vergangenen und gegenwärtigen, individuellen und kollektiven Lebens der Menschen, nicht zuletzt der in der Dichtung und in der Mythologie überlieferten, ihre aktuellste Kraft beziehen. Davon ausgehend geht es Müller, wie dem von ihm hochgeschätzten Walter Benjamin, dessen "Engel der Geschichte» er schon 1958 zum versteinerten "glücklosen Engel» umdichtete, um die «Auflösung der 'Mythologie' in den Geschichts-raum $»^{13}$. Der Blick auf eine solche heterogene Geschichte ist nicht dem «Sehzwang » unterworfen, der vom authentifizierten Dokument ausgeht, sondern eher dem «zunehmenden Druck authentischer Erfahrung», der, "vorausgesetzt, daß er "die Massen ergreift" ", die "Fähigkeit, der Geschichte ins Weiße im Auge zu sehen (entwikkelt) $»^{14}$. Dieses Weiße steht metaphorisch, für das, was sich in der Geschichte dem Dokument entzieht: z.B. die in breiten Kreisen der deutsch-deutschen Öffentlichkeit $\mathrm{zu}$ mythischen Gespenstern ausgewachsenen historischen Figuren Stalins und Hitlers (Szenen 2 und 4), was ihre historisierende Versachlichung durch unzählige Dokumente scheinbar nicht verhindern konnte.

Dies hat produktions- und rezeptionsästhetische Konsequenzen. Um diese komplexe Dramaturgie zu inszenieren, die Geschichte heißt, vertraut Müller in Germania 3 auf die Kraft mehrfach erprobter Mittel: u.a. der Dezentrierung und Fragmentierung von Fabel und Handlung - nicht zuletzt durch die auffallend zahlreichen Einlagen von fremden Textauszügen ${ }^{15}$-, oder der Gattungs-Entgrenzungen zwischen Epischem und Dramatischem ("Der Gastarbeiter»). Am wichtigsten aber sind vielleicht die systematischen Anachronismen, die "Telescopage der Vergangenheit durch die Gegenwart", aus der heraus, so W. Benjamin, durch Entstehung neuer Bild/SinnKonstellationen historische Erkenntnis «aufblitzen» und Aktualität neu definiert werden $\mathrm{kann}^{16}$. Jenseits aller Diskurse der immer zum Historismus neigenden Fachgeschichtsschreibung - aber nicht ohne Bezug zu ihnen, die mit souveräner Willkür in die Stücke eingewebt werden - stößt also Mythisch-Phantasmatisches immer wieder mit historisch Belegtem zusammen, damit schwer einzuordnende Handlungsund Denkmuster im sozialen Leben der Menschen durch die Konfrontation mit den in den Mythen buchstäblich verdichteten menschlichen Erfahrungen, wenn nicht erklärt, zumindest freigelegt werden können. Zwischen dem historisch verbürgten Geschehen und dessen vielschichtigen Reflexionen in alten und neuen Mythologien, vor allem den literarischen, schafft Müllers Theater rezeptionsästhetisch einen Raum der Repräsentation und Interpretation von Geschichte, der darauf vertraut, daß der so strukturierte dramatische Text mehr wissen kann als sein Autor ${ }^{17}$ und daß für den Leser/Zuschauer, dem viel " aufgepackt » wird ${ }^{18}$, dadurch ein neuer Raum subjektiver Produktivität entstehen kann. Insofern ist der Mythos, wie Müller 1988 formuliert, " ein Aggregat, eine Maschine, an die immer neue und andere Maschinen angeschlossen werden können. Er transportiert die Energie, bis die wachsende Beschleunigung den Kulturkreis sprengt. $»^{19}$. Dies kann am Beispiel eines besonders prägnanten Topos der Germania-Trilogie gezeigt werden. 
7 In Bezug auf seinen Philoktet (1966) bemerkte Müller, daß ein Vorgang in der mythologischen Handlung «andere, gleiche, ähnliche Vorgänge in der Geschichte » zitieren könne. So "zitier(e) der Kessel von Stalingrad Etzels Saal», wobei Müller ungewohnt vorsichtig hinzufügte: «Im Rückblick auf beide Ereignisse mag die Umkehrung einsichtiger sein $»^{20}$. Die Umkehrung ist in der Tat wichtig : sie macht den Unterschied zwischen dem «vernünftigen» und dem «schrecklichen» Mythos ${ }^{21}$, einem aufklärerischen und einem obskurantistischem Gebrauch von Mythologie deutlich. In Germania 3 zitiert Müller, wie in der Szene «Hommage à Stalin 1 » von Germania Tod in Berlin, einen berühmt-berüchtigten « Appell an die Wehrmacht » von Goering $^{22}$, der den « größten Heroenkampf unserer Geschichte » mit dem « Kampf der Nibelungen » gegen die Übermacht der Hunnen Attilas verglichen hatte. Hier verknüpft er sogar Goerings Phantasmagorien mit denen eines 1931 erschienenen, längst vergessenen Romans von F.C. Ettighoffer über den ersten Weltkrieg, Gespenster am Toten Mann (in BEA nachgedruckt), in dem u. a. geschildert wird, wie an diesem Ort bei Verdun (Le " Mort Homme ») ein wahnsinnig gewordener Offizier den Soldaten erzählt, "daß die Seelen der toten Soldaten noch lange nach den Schlachten in der Luft schweben und sich dort bekämpfen... ». Im ersten Germania-Stück hatte Müller gezeigt, wie der kleine Mann als Hitler-Soldat zum unbewußten Imitator des als Gespenst auftauchenden, mythisierten herrschenden Adels von einst (Nibelungen) und zum Kannibalen seinesgleichen wurde. In Germania 3 beschreibt er bei den StalingradSoldaten die Wiederkehr des gleichen Nibelungen-Kannibalismus mit kanonisch gewordenen Formeln des Grauens («Esst eure Toten und löscht euren Durst / Mit ihrem Blut...», S. 27). Damit werden die elenden «Wiederkehrer » als reale Wiedergänger des vormals literarischen Grauens im Dienste der mythenbeschwörenden nationalsozialistischen Machthaber gezeigt. Die Toten von Stalingrad werden dann selbst zu ewigen Gespenstern, die in den Köpfen unzähliger Generationen herumspuken werden.

8 Solche mythopolitischen Topoï, die machtmanipulierte Wiederholungszwänge inszenieren, sind Müllers typische dramaturgische Vorgehensweise, das wahrhaft Gespensterhafte an der deutschen Geschichte aufzudecken. Müllers Fazit: "Die deutschen Soldaten haben im Kessel von Stalingrad die Lektion der Nibelungen nicht gelernt. Die wiederholte Einmaligkeit muß mit zitiert werden. Erst wenn das Modell geändert wird, kann aus der Geschichte gelernt werden ${ }^{23}$. »

9 Einen solchen Versuch, dieser Macht der mythologischen Fiktion als Macht der Gespenster über die Lebenden ein (ebenfalls fiktionales) Ende zu setzen, wird jedoch in der dritten Szene von Germania 3 von einer (doppelten) literarischen Fiktion - als Intertext Hebbel/Müller - unterbrochen : die umfunktionierte Hebbelsche Kriemhild stellt sich als rachedurstige Rotarmistin dem Kampf mit dem adeligen deutschen Hitler-General Hagen. Dadurch durchbricht sie den Teufelskreis der ewigen Wiederkehr des Gleichen, gibt aber möglicherweise - gleichsam als neue Allegorie der «Dialektik der Aufklärung» - zugleich den Weg frei für weitere antifaschistische Nachkriegsmythen. Diese, wie man allerdings erst seit der Öffnung der Archive genauer weiß, können zu neuen Gründungsmythen avancieren, die eine neue Staatsmacht legitimieren ${ }^{24}$. 


\section{Ein Gespenst geht nicht mehr um, nur noch Gespenster $^{25}$}

10 Müller, der noch 1987 befand : «Wir haben keinen anderen Weg nach vorn als zurück zu Marx und Lenin ${ }^{26}$, nimmt in Germania 3 den Anfang des Kommunistischen Manifestes durchaus ernst. Wenn der Kommunismus überhaupt noch « umgeht » in Europa, dann höchstens als Gespenst. 1971 hatte das zweite Germania-Stück noch mit einer ambivalenten Allegorie der sozialistischen Hoffnung geendet : ein sterbender Maurer und Altkommunist verwechselt in seinem Todesdelirium eine « junge Hure » mit der « roten Rosa » (Luxemburg), während ein junger Maurer, um diese Illusion nicht zu zerstören, dem Mädchen stereotype Zukunftsvisionen (« die roten Fahnen über Rhein und Ruhr ») souffliert. Germania 3 kann nur noch das selbstverschuldete - und von Müller wohl nie verwundene - allgemeine Scheitern des Sozialismus/Kommunismus registrieren: ablesbar schon an der Eingangsszene, in der die Geister Ulbrichts und Thälmanns als Grenzwachtposten ihren tödlichen Dienst an der Berliner Mauer mit zynischer Ratlosigkeit verrichten, während Rosa Luxemburg in Begleitung ihrer Mörder stumm vorbeigeht. Dabei spielt diese Ikone eines andersgearteten Sozialismus eine Rolle, die nur aus ihrem intertextuellen Platz im Werk Müllers und an bestimmten Text-Disseminationen in Germania 3 (das "Jägerlied») zu erschließen ist, wobei das Stück als Moment eines mehrdimensionalen Intertextes erscheint. Darauf näher einzugehen, ermöglicht eine konkretere Einsicht in die sehr komplexe Müllersche Fabrikation literarischer Geschichtsfiktionen.

11 Als Rosa Luxemburg gleich nach der letzten Replik der ersten Szene in Begleitung ihrer Mörder buchstäblich « erscheint », während das Lied «Es blies ein Jäger wohl in sein Horn und alles was es blies war verloren » ertönt, kann dieses als Leitmotiv des einen Mörders, des "Jägers Runge », verstanden werden. Dieses verfremdende, unerwartete Assoziationen auslösende Leitmotiv ist am Ende der Stalingrad/Nibelungen-Szene wieder zu hören, als Kriemhild ihren Mantel ablegt und in der Uniform eines einfachen Soldaten der Roten Armee ihren Fluch auf Siegfrieds Mörder ausspricht, der in einer « deutschen Generalsuniform » erscheint. Von hier aus läßt sich ein weiterer Bezug zu einer Stelle des Textes «Die Wunde Woyzeck» herstellen, der den Titel der Szene «Siegfried eine Jüdin aus Polen » enträtselt. Darin wird Runge als Woyzecks «blutiger Bruder» und "proletarisches Werkzeug der Mörder von Rosa Luxemburg» bezeichnet : " sein Gefängnis heißt Stalingrad, wo die Ermordete ihm in der Maske der Kriemhild entgegentritt [...] ihr deutsches Monument, die Mauer, in Berlin... ${ }^{27}$. Im Bedeutungsnetz des Intertextes verschmelzen also Täter (Hagen/Runge) und Opfer (Siegfried/R. Luxemburg als "Jüdin aus Polen») zu einer verdichteten hybriden Gestalt. Eine weitere Assoziationsreihe, die der historischen Bedeutung R. Luxemburgs und ihrer Ermordung eine viel größere Tragweite verleiht, ohne die Konfrontation von Geschichte und Mythos aufzugeben, bietet das Darmstädter Müller-Interview von 1986, «Ich bin ein Neger » : «Die Rosa Luxemburg war ein [...] kontroverser Dialogpartner [...] für Lenin. Danach gab es keinen Partner mehr, es gab nur noch Funktionäre, und das war [...] ein europäisches Verhängnis. Und ob sie das wußten oder nicht, diese Offiziere haben einen historischen Auftrag vollzogen, die die (Rosa Luxemburg) umgebracht haben. Sie haben die deutsche Revolution enthauptet und damit in Abhängigkeit gebracht von der russischen und damit eigentlich den Hitler vorbereitet. » Insofern, so Müller weiter, sei die Berliner Mauer « ein Denkmal für Rosa 
Luxemburg, positiv und negativ » und die «DDR-Partei » eine «besiegte Partei, wie Kriemhild eine besiegte Partei war, die eben den Attila geheiratet hat, um sich zu rächen an den Mördern von Siegfried ${ }^{28}$. » Der « Kessel von Stalingrad » zitiert dann nicht nur «Etzels Saal », sondern destilliert aus der Konfrontation von Geschichte und Mythologie eine so faszinierende wie schwer lesbare « Wiederkehr des Gleichen » vor dem Hintergrund von Klassenkämpfen und gescheiterten deutschen Revolutionen heraus. Daß darüber hinaus Müllers subjektive Einstellung zur Hauptgestalt eines für die DDR-Machthaber gespenstisch gewordenen Sozialismus ein zusätzliches Licht auf die erste Szene von Germania 3 werfen kann, läßt sich einem Le Monde-Beitrag Müllers vom März 1979 entnehmen: "Aus dem verwüsteten Frankfurt über die Vitrine Westberlin heimgekehrt in das trübe Licht am Bahnhof Friedrichstraße, bin ich froh, daß Rosa Luxemburg, Jüdin aus Polen, Revolutionärin in Deutschland, auf dieser Seite der Mauer begraben liegt ${ }^{29}$. » Auf die Situation von 1990 projiziert : die Schutzpatronin eines zunächst «falsch gemachten" und dann nur noch «real» existierenden Sozialismus wird, wenn es kein Diesseits und Jenseits der Mauer mehr gibt, zum ewigen Gespenst.

\section{Erzählungen : große und kleine}

Einen paradoxen Vorteil bietet Müllers zufolge die deutsche Wiedervereinigung: der Dichterblick kann ungetrübt auf dem Höhepungt der deutschen Katastrophen, dem zweiten Weltkrieg, und auf dessen Hauptprotagonisten länger verweilen. "Mich interessiert, was Deutschland betrifft, der Zweite Weltkrieg. Jetzt ist es möglich, Hitler und Stalin in Beziehung zu setzen, auch im Theater. Die beiden können jetzt miteinander reden, ihre Arbeit ist getan ", erklärt Müller $1992^{30}$. Deshalb gebührt ihnen in der dramaturgischen und diskursiven Ökonomie von Germania 3 ein bevorzugter Platz. Sie führen als Gespenster miteinander und mit anderen Gespenstern (Lenin, Trotzki, Goebbels) einen Dialog, der aus langen Monologen besteht, da sie als einsame Diktatoren, wie Hamlet, «keinen Partner " haben ${ }^{31}$. Was Hitler und Stalin zunächst verbindet, wie bei Shakespeares Richard III oder Macbeth, ist die einsame Macht und einsame Nacht ${ }^{32}$ des Monologierens, die nur noch tote Adressaten zuläßt, denen keine Widerrede zugebilligt wird. Das rückt sie anscheinend in die Nähe ihrer Antithese, des « rosa Riesen » der Schlußszene, der « mit keinem rede(t), der nicht tot ist» (S. 80). Aber nur scheinbar. Denn der «rosa Riese» ist ein ebenfalls monologisierender Repräsentant derjenigen in Germania 3, die nicht gegen, sondern neben den Trägern der " großen Erzählungen », im Sinne Lyotards - hier vor allem Nazismus und Stalinismus -, ihre «kleinen» Erzählungen zu ihrem Recht kommen lassen. Weshalb diese Erzählungen, die zu den "großen» einen impliziten Kontrapunkt bilden, unterschwellig mit diesen dialogieren. Es sind kurze Chroniken eines «beschädigten Lebens » (Adorno), weit unterhalb der großen Geschichte aber in enger Verbindung mit ihr, in denen seelisch-körperliche Wurzeln der ewigen Wiederkehr des (barbarisch) Gleichen durchscheinen. Der " rosa Riese », der russische Soldat und ehemaliger GulagHäftling der dritten Szene (S.17-18), der vormals kommunistische und zum HitlerAnhänger gewordene deutsche Soldat im 2. Teil derselben Szene (S.22-23), der in zweierlei Gestalt mordende Kroate (Sz. 5, S. 40-42), alle berichten über das katastrophale Erleben der Geschichte von unten, in der Täter und Opfer eine pervertierte Dialektik des Grausamen vorführen. So beim russischen Soldaten : « ... Das war der Gulag / Mein Glück sind die Deutschen / Weil Russland ist mehr als der Gulag 
und / Stalin bricht Hitler das Genick mit unsern / Dreimal von ihm gebrochenen Knochen " (S. 18) ${ }^{33}$. Ähnlich im Bericht des ehemaligen Kommunisten, in dem die Kontinuität zwischen der KPD von 1928 und der stalinisierten SED angedeutet und eine mögliche Antwort auf Thälmanns « Frage » in der ersten Szene (« Was haben wir falsch gemacht.») gegeben wird: erzählt wird nämlich, wie Kommunisten ein siebzehnjähriges Parteimitglied, das "auf die schiefe Bahn geraten (war) », bei der Polizei abliefern («Und schleppen unseresgleichen auf die Schlachtbank»), um kurz vor den Wahlen die Chancen der Partei nicht zu gefährden : «Für unsere Sitze in der Stadtverwaltung / Wir haben auch das Kopfgeld noch kassiert / Fünfhundert Reichsmark für die Rote Hilfe » (S. 23). Einen schlüssigen Kommentar dazu liefert ein in BEA auszugsweise abgedruckter Text von A. Simon, « Versuch, mir und anderen meine ostdeutsche Moral zu erklären» (1993), über die herrschende "nachfaschistische Moral» der DDR, «eine unter brutalen Umständen und im Widerstand gegen Unmenschlichkeit entstandene Moral, die selbst wieder brutal war $»^{34}$.

Diese « kleinen Erzählungen », die so viel aussagen über das Leiden der kleinen Leute an der Geschichte und die sich daraus ergebenden Irrungen und Wirrungen lesen sich wie chaotische Bruchstücke einer Mentalitätsgeschichte von unten, die die fast vollständig zitierte Erzählung des "kleinen Mönchs » aus Galilei in exemplarischer Form darlegt. Die Brechtsche Erzählung ist von Müller sicherlich weniger als Gelegenheit eines ideologischen Umfunktionierens der ursprünglichen Intention Brechts, d.h. als Parteiergreifung für den kleinen Mönch gegen Galilei gedacht, wie der fiktionale Palitzsch es tut (S. 54). Sie erhält im neuen Kontext zwar einen anderen, im «Hypertext» aber potentiell vorhandenen Sinn: den einer ernstzunehmenden Reaktion der kleinen Bauern auf die "große Erzählung» der galileischen Theorie, die zunächst eben nur theoretisch die Tore des menschlichen Fortschritts öffnet. "Nein ich sehe ihre Blicke scheu werden [...], ich sehe, wie sie sich betrogen und verraten fühlen. Es liegt also kein Auge auf uns, sagen sie. Wir müssen nach uns selber sehen, ungelehrt, alt und verbraucht wie wir sind ? [...] Kein Sinn liegt in unserem Elend, Hunger ist eben Nichtgegessenhaben... » (S. 55). Inszeniert wird hier diese Angst vor dem Neuen und dessen möglichen Schrecken, die auch Hoffnung einschließt und die Müller aus Rilkes zweiter «Duineser Elegie » - und im dialektischen Geiste Brechts - auf eine bei ihm leitmotivisch wiederkehrende Formel gebracht hat. «Damit etwas kommt muss etwas gehen die erste Gestalt der Hoffnung ist die Furcht die erste Erscheinung des Neuen der Schrecken $»^{35}$.

Emblematische Figur aller Unterdrückten, Geschundenen und Ent-rechteten, die als Mörder an der allgemeinen Barbarei teilhaben, ist in der politisch-literarischen Mythologie Müllers der Büchnersche Woyzeck, der als «die Wunde Woyzeck» im gleichnamigen Text von 1985 irgendwo als " Hund begraben liegt », während "wir » auf seine "Auferstehung " als Wolf, d.h. auf den von den "Verdammten dieser Erde » ausgelösten revolutionären Aufstand, «mit Furcht und/oder Hoffnung " warten ${ }^{36}$. In Germania 3 ist die Ambivalenz Furcht/Hoffnung - etwa im Unterschied zu Der Auftrag der bloßen Wiederholung des Furchterregenden gewichen. Die Wiederkehr des Furchtbaren als einzige Zukunftshoffnung, seitdem der internationale Kommunismus diese Funfkion im Imaginären der Ausgebeuteten nicht mehr erfüllt, setzt bei diesen Energien frei, die sich der Nazismus seinerzeit schon zunutze gemacht hatte. Ausgehend von der Tatsache der soziologisch proletarischen und ideologisch kommunistischen Herkunft manchen SA-Manns erklärt Müller mit sichtbarer Lust am 
provozierenden Paradox in einem zur Zeit der Arbeit an Germania 3 gegebenen Interview: "Dadurch ist ein Großteil linker Energie von den Nazis aufgenommen worden. [...] Der Nationalsozialismus war eigentlich die größte historische Leistung der deutschen Arbeiterklasse. Der Blitzkrieg [...] war [...] die Herausführung der deutschen Arbeiterklasse aus dem Status der Ausgebeuteten in den Jägerstatus. [...] Der Krieg wurde zum Ersatz für die Revolution, genauso wie jetzt die Krawallen gegen Ausländer wieder der Ersatz sind für die nicht zu Ende geführte Revolution. »

Auch der sadistisch veranlagte "rosa Riese» der Schlußszene, der als «Tod von Brandenburg " in den 1990er Jahren die DDR terrorisierte, verkörpert eine perverse Asozialität, die im Gegensatz zum Brechtschen Fatzer - einem Fragment, das Müller bewunderte und gleich $\mathrm{zu}$ Beginn seiner Intendantenzeit am Berliner Ensemble inszenierte -, nicht mehr produktiv, sondern nur noch sinnlos destruktiv und selbstdestruktiv ist. Seine Kleidungsattribute - der rosa Unterrock seiner von den Russen vergewaltigten toten Mutter und die Jacke seiner "zweiten Mutter », der Armee - stilisieren ihn zu einer Allegorie des Grotesken, des schizoiden kleinen Mannes, dessen tiefsinnig-chaotische Sprache zusammenhanglose Formeln aus deutschen Märchen mit der Beschreibung dessen vermischt, was ihn seelisch zerstört hat. Und der in Stalingrad von Russen getötete junge deutsche Soldat, der Hölderlins Empedokles zusammen mit dem Photo von am Galgen hängenden Partisanen in seinem Stiefel aufbewahrt (Sz. 3, Teil I, ), ist ein anderer Vertreter der barbarisch mordenden proletoiden Existenzen, die im Zeichen der allgemein herrschenden Barbarei beides in einem sind: unterwürfige Hunde des herrschenden gesellschaftlichen Systems, das sie ausbeutet und verknechtet, und sadistisch wütende Wölfe, die sich vorzugweise an ihresgleichen vergreifen. Wie die asia-tischen «Wölfe », die in Stalins Lagern auf ihren blutigen Einsatz in Hitler-Deutschland gewartet haben (« Weil sie dich hassen, werden sie mich lieben - S.13), oder die Jugendbanden in den schwarzen Ghettos des hochkapitalistischen New Yorks, machen sie alle deutlich, « was Marx vergessen oder nicht gewußt hat: die Gewalt des Tribalismus $»^{37}$, die in Müllers Augen den zu zahlenden Preis für ausgebliebene Revolutionen ist.

\section{Dialog mit toten Dichtern}

16 Weil eine «Funktion von Drama Totenbeschwörung (ist) », darf der « Dialog mit den Toten nicht abreißen, bis sie herausgeben, was an Zukunft mit ihnen begraben worden ist $»^{38}$. So beschwört Müller weitere Gespenster herauf, tote Dichter, aus deren Werken er Blöcke heraussprengt, die wiederum als produktive Fremdkörper fungieren. Sie sprengen das Kontinuum des Müllerschen Textes, verdunkeln und erhellen zugleich diesen "Wirtstext », adressieren ihn neu, indem sie Stil- und Sinnbrüche zur Grundlage einer neuen Lektüre machen. Es sind, theaterbegrifflich gesprochen, weder Hauptnoch Nebentexte, sondern Zwischentexte, die überdies den eigenen Texten als vergangenen, so bei den Zitaten aus Müllers Philoktet (S. 76) und Macbeth (S. 64), durch Einbettung in einen neuen Kontext zu einer sinnvollen Aktualisierung verhelfen. Sie können beispielsweise eine Art Parabelfunktion erfüllen, für die Kafkas Das Stadtwappen, in der Szene " Party ", als Modell dienen kann und deswegen hier näher beschrieben werden soll. In dieser Szene diskutieren prominente Vertreter des pragmatisch, zynisch und dekadent gewordenen DDR-Sozialismus stalinistischer Observanz über sozialistische Politik und Wohnungsbaupolitik, während im Radio die 
große Rede Chruschtschows vor dem XX. Parteitag der KPdSU ertönt, die die «Entstalinisierung » einleitete. Hier zeigt sich, daß die eingeschobene Kafka-Parabel « geräumiger ist, mehr Realität aufnehmen kann (und mehr hergibt) als die Parabel Brechts», da sie "nicht orientiert auf eine Bewegung (Praxis), auf eine Bedeutung nicht reduzierbar (ist), eher fremd als verfremdend, ohne Moral ${ }^{39}$. » Kafkas Erzählung handelt nämlich vom babylonischen Turmbau, der im Szenenkontext zur Metapher des utopischen sozialistischen Aufbaus wird : «Das Wesentliche des ganzen Unternehmens ist der Gedanke, einen bis in den Himmel reichenden Turm zu bauen. [...] Der Gedanke, einmal in seiner Größe gefaßt, kann nicht mehr verschwinden; solange es Menschen gibt, wird auch der starke Wunsch da sein, den Turm zu Ende zu bauen. »

Ein weiterer Topos der Kafkaschen Erzählung, der sich ohne weiteres auf den « realexistierenden Sozialismus » übertragen läßt, ist, daß die Menschen sich langsam klarmachten, daß der Turmbau die Arbeit mehrerer Generationen erfordern würde und infolgedessen das langfristige Ziel zugunsten eines kurzfristigeren, des «Baus einer Arbeiterstadt ", langsam aus den Augen verloren. Daß die Turmerbauer in ihren "Sagen und Liedern" dennoch "von der Sehnsucht nach einem prophezeiten Tag (erfüllt waren), an welchem die Stadt von einer Riesenfaust in fünf kurz aufeinanderfolgenden Schlägen zerschmettert werden wird», ist die mythische Antwort auf die wohnungsbauliche Realität des DDR-Sozialismus, in dem die «Arbeiterstädte» nur «Arbeiterschließfächer» (S. 66) bzw. "Fickzellen mit Fernheizung von Rostock bis Johanngeorgenstadt » (S. 7) sind.

\section{Der zweite Tod des Bertolt Brecht : « Wenn keine andere Wahl ist, ziehe ich den Kannibalismus der Lebenden dem Vampirismus der Toten vor. " ${ }^{40}$}

In solchen Dialogen mit toten Dichtern wird Brecht ein Sonderstatus zuerkannt. In der 7. Szene (« Maßnahme $1956 »)$ inszeniert Müller eine mehrschichtige « Telescopage von Vergangenheit durch die Gegenwart ", die Aktualität wieder über Anachronismen erzeugt und gleichzeitig das Gespensterhafte am jüngst verstorbenen «Stückeschreiber » herausstellt. Er zitiert zunächst Brechts umstrittenstes Lehrstück im Szenentitel und deutet es in der Handlung «en abyme » um : der realexistierende DDR-Bildhauer Fritz Cremer habe "vergessen, masszunehmen" und müsse wissen, «ob die Grösse stimmt» (S. 60). Das tut auch Müller durch einen von gleich zu gleich geführten, intertextuellen Dialog mit dem toten Brecht, der bis dahin in den Repliken seiner Schüler und seiner Witwen als gespenstisch wirkende Autorität des Hauses herumspukte. Das Fortschreiten der Geschichte wird durch die réécriture des Brechtschen Gedichts «Ich benötige keinen Grabstein» in einem zutiefst pessimistischen Sinne indiziert. Indem die ursprüngliche Aussage durch den Zusatz einer einfachen Negation den aktuellsten Stand (1995) der Brecht-Rezeption dokumentiert ("Er hat Vorschläge gemacht / Wir haben sie nicht angenommen »), macht sie den Weg frei für ein weiteres Eindringen Müllers in den fremden Text, das nun ihm selbst gilt, wie die genuin Müllersche Ausdrucksweise beweist: "Warum sollten wir / Und das soll stehn auf meinem Grabstein und / Die Vögel sollen darauf scheißen und / Das Gras soll wachsen über meinen Namen / Der auf dem Grabstein steht Vergessen sein / Will ich von allen eine Spur im Sand.» (S. 64-65). Der Intertext entsteht durch die Fiktion einer umgeschriebenen Grabschrift, als würden hier ein 
Toter auf Abruf mit einem wirklichen Toten den einzig möglichen Dichterdialog fortführen wollen. Die weiter oben zitierte Müller-Stelle ist insofern eine ernstzunehmende Metapher: Müllers Kannibalismus, die Einverleibung des Brechtschen Gedichts als dessen "Aufhebung ", soll eins verhindern, das Müller auch für sich befürchtet haben mag: den Vampirismus des toten Brecht, dem seine gralshütenden Witwen in seinem Theater auf hilflose Weise Vorschub leisten. Nun ist möglicherweise Müller selbst zum vampirähnlichen Gespenst geworden, das eine Grabschrift dringend benötigt. Auch wenn seine Stücke auf vielen Bühnen inszeniert wurden, sind sie im Gegensatz zu den Brechtschen nicht mehr eindeutig " adressiert ». Es sind grundsätzlich « einsame Texte, die auf Geschichte warten ». Sie erheben aber nach wie vor den Anspruch, gegen den «Imperialismus der Besetzung von Phantasie und der Abtötung von Phantasie durch die vorfabrizierten Klischees und Standards der Medien» - was nicht zuletzt für die politischen gibt - eine "primär politische Aufgabe » zu erfüllen. Es gilt, mit den Mitteln der Kunst « die Wirklichkeit unmöglich zu machen ${ }^{41}$.» Eine Wirklichkeit, die Müller wiederum nur mit Hilfe antiker Mythen zum Ausdruck bringen konnte: "Im Jahrhundert des Orest und der Elektra, das heraufkommt, wird Ödipus eine Komödie $\operatorname{sein}^{42}$. »

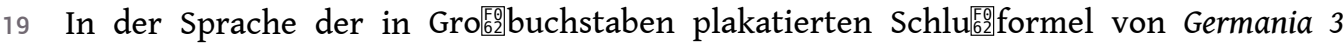
ausgedrückt, die eine optimistische Replik aus Brechts Galilei abwandelt: [DUNKEL GENOSSEN IST DER WELTRAUM SEHR DUNKEL.]

\section{NOTES}

1. Vgl. H. Müller, Germania 3 Gespenster am Toten Mann, Köln 1996. Das Stück ist in 9 Szenen aufgegliedert. Sie seien hier zur besseren Orientierung kurz beschrieben :

1) «Nächtliche Heerschau» (Thälmann und Ulbricht an der Berliner Mauer 1961) - 2) «Panzerschlacht» (Stalin zur Zeit des deutschen Überfalls auf die UdSSR im Juni 1941) - 3) «Siegfried eine Jüdin aus Polen» (Schlacht von Stalingrad im Winter 1942/43) 4) «Es blies ein Jäger wohl in sein Horn» (Hitler kurz vor seinem Selbstmord am 1. Mai 1945) - 5) "Der Gastarbeiter » (3 Teilszenen : zwischen April 1945 und 1990) - 6) «Die zweite Epiphanie » (Nach dem deutschen Zusammenbruch erschlägt ein heimkehrender kommunistischer KZ-Häftling einen russischen Soldaten, der seine Frau vergewaltigt, und wird in den Gulag verschleppt, wo er niedergeschlagen wird) - 7) «Maßnahme 1956 » (Berliner Ensemble: Nach Brechts Tod im August 1956 proben seine beiden Hauptschüler M. Wekwerth und P. Palitzsch Brechts Bearbeitung von Shakespeares Coriolan, während drei « Brecht-Witwen » - H. Weigel, E. Hauptmann und I. Kilian eine Art Parallelhandlung führen) - 8) « Party » (Während einer Feier mit SED-Funktionären und Vertretern der Intelligenz wird die Rede Chruschtschows vor dem XX. Parteitag der KPdSU vom Februar 1956, die die «Entstalinisierung » einleitete, im Radio gehört) - 9) «Der rosa Riese » (Monolog eines Serienmörders, der 1990 die Bevölkerung Brandeburgs in Schrecken versetzte).

In einer vom Berliner Ensemble herausgegebenen Auswahl verschiedener Texte zur Spielzeit 1995/96 (Berlin o.J. ), als Germania 3 aufgeführt wurde, finden sich zahlreiche Auszüge aus Werken, die den größten Teil des in Germania 3 verarbeiteten kulturellen Intertextes ausmachen : literarische Texte (auch von Müller selbst), Dokumente, Berichte, historische Darstellungen, 
kulturphilosophische Traktate, Interviews und Gespräche (auch mit Müller selbst) und eine relativ umfangreiche Ikonographie, die diesen Intertext um eine wesentliche visuelle Dimension erweitert. Ich werde des öfteren daraus zitieren (= BEA, ohne Seitenzahlangabe). Ich verweise ebenfalls auf die 1996 vom Berliner Ensemble herausgegebene Drucksache 20, mit Materialien zur Martin Wuttke-Inszenierung von Germania 3, u.a. Auszügen aus dem Arbeitsbuch von Müller und M. Lammert, der Bühnenbild und Kostüme entwarf.

2. Vgl. Drucksache 20, a.a.O., S. 831.

3. G. Schulz, Heiner Müller, Stuttgart 1980, S. 129.

4. Im selben Jahr schreibt Heidegger : «Der Streit ist kein Riß als das Aufreißen einer großen Kluft, sondern der Streit ist die Innigkeit des Sichzugehörens der Streitenden.» (Holzwege, Frankfurt a. M.1950, S.51). Ist dieses von Müller immer wieder beschworene Motiv die aktualisierte Wiederaufnahme eines philosophischen Diskurses, in dem die politische Dimension der deutschen Teilung in einem anthropologischen Mythos aufgelöst wurde?

5. Vgl. dazu die 1977 erschienene Groteske Leben Gundlings Friedrich von Preußen Lessings Traum Schlaf Schrei, die die autoritäre Volksdisziplinierung und die Servilität der Intellektuellen bzw. deren ohnmächtiges Opponieren im friederizianischen Preußen als Vorgriff auf den späteren «preußischen Sozialismus » inszenierte.

6. S. dazu F.M. Raddatz, Dämonen unterm roten Stern, Stuttgart 1991, S. $56 f$.

7. « Mülheimer Rede» (1979), in : F. Hörnigk (Hg.), Heiner Müller Material, Leipzig 1989, (= HMM), S. 101.

8. Eine Art interdiskursiven Nebentext zu Germania 3, der die ideologische Seite der Teilung kommentiert, stellt der « lexikalische Anhang » zum Stück dar. Dort werden u.a. die wichtigsten darin vorkommenden Ereignisse und Gestalten anhand von Stichworten aus einer DDREnzyklopädie von 1957 und einem BRD-Lexikon von 1955 synoptisch als definitorische Embleme deutsch-deutscher Ideologien herausgestellt.

9. In seiner Autobiographie schreibt Müller: «Der Beweis für die Existenz der DDR [...], war zuletzt nur noch die Grenze, die Toten an der Mauer, dem Mausoleum nicht nur des deutschen Sozialismus, ihre letzte perverse Legitimation. " (Krieg ohne Schlachten Leben in zwei Diktaturen, Köln 1992, S. 349).

10. Diese Szene war in einer anderen Fassung ursprünglich 1971 für Germania Tod in Berlin geschrieben worden und sollte bei der Aufführung des Stücks im Januar 1989 am Berliner Ensemble gezeigt werden, wurde es aber aus politischen Gründen nicht.

11. S. Wirsing : «Der Ausgang der Geschichte », in : Theater heute, H.8, August 1996, S.34.

12. «Ein Brief », in : HMM, S. 37.

13. Müllers Text wurde wiederabgedruckt in HMM, S. 7. Das Benjamin-Zitat befindet sich in : Das Passagen-Werk, Bd. I, S. 571.

14. « Fatzer \pm Keuner », in : HMM, S. 31.

15. Es handelt sich um Hölderlins Empedokles (Sz. 3), Kleists Prinz von Homburg (ebd.), Hebbels Nibelungen (ebd. ), Grillparzers Die Ahnfrau (Sz. 5), Brechts Galilei und Coriolan (Sz. 7), Kafkas Das Stadtwappen (Sz. 8).

16. Vgl. Das Passagen-Werk, a.a.O., S. 588.

17. Vgl. KoS, S. 257.

18. In einem Gespräch von 1975 erklärte Müller : «Ich habe, wenn ich schreibe, immer nur das Bedürfnis, den Leuten so viel aufzupacken, daß sie nicht wissen, was sie zuerst tragen sollen, und ich glaube, das ist auch die einzige Möglichkeit. » In : Sonderheft von Theater heute, Velber bei Hannover 1975, S. 121.

19. «Shakespeare eine Differenz », in : HMM, S. 106.

20. «Drei Punkte. Zu Philoktet », in : HMM, S. 61. In einem Interview von 1992 erklärte Müller : «Und der Kessel von Stalingrad ist der Sarg von Attila. Der einzig nationale Stoff sind die Nibelungen. » in : Drucksache 16, Berliner Ensemble, Berlin 1995. 
21. Vgl. W. Emmerichs Aufsatz in : HMM, S. 138-156.

22. Der Appell wurde im Völkischen Beobachter vom 2.2.1943 veröffentlicht und in BEA auszugsweise abgedruckt.

23. « Drei Punkte. Zu Philoktet », a.a. O., S. 61.

24. Wie verhängnisvoll der Stalingrad-Topos für die Geschichte der DDR sein konnte, unterstreicht Müller in einem Freitag-Gespräch vom 18.6.1993, in dem er in einer kühnen Paradoxie den Modellcharakter der emblematischen Schlacht des zweiten Weltkriegs auch für den sozialistischen Frieden betonte. Das Modell der DDR sei « eigentlich Stalingrad (gewesen)». Das bezeuge « die Bildung der Ostblockstaaten als gefrorene Kessel : Abgrenzung nach außen, Zerstörung der Binnenstruktur. »

25. Ende 1989 verfaßte Müller Gedichte über die Ereignisse in der DDR, die mit Photographien des 1986 in Ost-Berlin aufgestellten Marx-Engels-Denkmals unter dem Titel «Ein Gespenst verläßt Europa » (Köln, 1990) publiziert wurden.

26. «Berlin, ein Ort für den Frieden », in : HMM, S. 112.

27. Ebd., S. 114 (auch in BEA abgedruckt).

28. Zit. nach BEA.

29. Abgedruckt in : HMM, S. 91.

30. Vgl. KoS, a.a.O., S. 257.

31. So erklärt Müller die «Dominanz der Monologe » in Shakespeares Stück (vgl. « Shakespeare eine Differenz », in : HMM, S. 106).

32. Vgl. den Anfang von Stalins Tirade (S.9) : «Genosse, warum trinkst du in der Nacht; / Was fürchtest du, wo deine Macht Gesetz ist. »

33. Diese Ansichten decken sie mit denen Stalins (S. 13), mit dem ein unmöglicher Opfer/HenkerDialog geführt wird: «Hitler, mein Freund von gestern. Bruder Hitler. / Verbrennst du meine Dörfer. das ist gut. / Weil sie dich hassen, werden sie mich lieben. / Deine Blutspur wäscht meinen Namen weiß. /... / Mein Rücken heißt Asien, meine Wölfe warten / Das haben sie gelernt in meinen Lagern. »

Dieser Topos steht in einem intertextuellen Bezug zu einem Roman von W. Grossmann, Leben und Schicksal (Frankfurt a. M. / Berlin 1987) : «Er (Stalin) stellte sich vor, daß im Staub hinter Hitlers Panzer, im Qualm, all diejenigen schritten, die er scheinbar auf immer und ewig für unterworfen, bestraft, zur Ruhe gebracht gehalten hatte. Sie krochen aus der Tundra, durchbrachen den ewigen Bodenfrost, unter dem sie lagen, zerfetzten den Stacheldraht. » (zit. nach BEA).

34. Der Text wurde im Kursbuch 111, Feb. 1993, veröffentlicht.

35. Zuerst in der « Anmerkung " zu Mauser (1970). Vgl. auch «Fatzer + Keuner », a.a.O., S. 36, wo die Formel auf Marx übertragen wird.

36. Vgl. HMM, S. 115. In BEA wieder abgedruckt.

37. « New York oder Das eiserne Gesicht der Freiheit », in : HMM, S. 98.

38. H. M., Gesammelte Irrtümer, S. 64. Ich verweise hier auf meinen Müller-Aufsatz in : Germanica $14 / 1994$, S. $207 f$.

39. « Fatzer + Keuner », a.a.O., S. 31.

40. «Und vieles / Wie auf den Schultern eine / Last von Scheitern ist / $\mathrm{Zu}$ behalten... (Hölderlin) », in : HMM, S. 92.

41. Alle Zitate aus verschiedenen Texten in : HMM, S. 24, 63, 40, 28, 94.

42. « Projektion 1975 », in : H.M., Die Umsiedlerin oder das Leben auf dem Lande, Texte 3, Berlin 1975, S.117. 


\section{RÉSUMÉS}

Heiner Müllers unwiderruflich letztes Stück, Germania 3 Gespenster am Toten Mann, nutzt die deutsche Wiedervereinigung von 1990 als Gelegenheit aus, um deren komplexe Vorgeschichte im Zeichen des unheilvollen Germania-Mythos zu dekonstruieren. Er inszeniert sie also nicht, oder kaum, als solche, sondern eher als peripheres Ereignis, das die bisherige deutsche Geschichte als Wiederkehr der gleichen Katastrophen nicht wirklich unterbricht. Dazu beschwört er eine Reihe von politischen und literarischen, berühmten und unbekannten Gespenstern herauf, die Mythologie und Geschichte - insbesondere die des deutschen Sozialismus - zum Zweck eines besseren Verständnisses dieses unseres « dunklen Weltraums » miteinander kollidieren lassen.

Germania 3 Gespenster am Toten Mann, qui restera la dernière pièce de Heiner Müller, se sert de la réunification allemande de 1990 pour en déconstruire une préhistoire placée sous le signe fatal d'une mythique Germanie. Il ne la met donc quasiment pas en scène comme telle mais en fait plutôt un événement marginal qui n'interrompt pas vraiment le cours de l'histoire allemande conçue ici comme éternel retour des mêmes catastrophes. Pour cela, il convoque toute une série de fantômes, politiques ou littéraires, inconnus ou célèbres, qui font s'entrechoquer mythologie et histoire - en particulier celles du socialisme allemand - afin de permettre une meilleure compréhension du « sombre univers » qui est le nôtre.

\section{AUTEUR}

\section{ANDRÉ COMBES}

Université Charles-de-Gaulle-Lille 3 